Revue des patrimoines et plasticiens (XXe-XXle siècles)

\title{
Des archives de Jean-Claude Dondel aux archives de l'administration : des sources qui renseignent sur les collaborations entre architectes et artistes
}

The archives of Jean-Claude Dondel and the archives of a public administration, sources illustrating the question of the collaboration between architects and artists

\section{Anaïs Carré}

\section{(2) OpenEdition}

\section{Journals}

Édition électronique

URL : http://journals.openedition.org/insitu/14639

DOI : $10.4000 /$ insitu. 74639

ISSN : 1630-7305

Éditeur

Ministère de la culture

Référence électronique

Anaïs Carré, «Des archives de Jean-Claude Dondel aux archives de l'administration : des sources qui renseignent sur les collaborations entre architectes et artistes », In Situ [En ligne], 32 | 2017, mis en ligne le 27 juillet 2017, consulté le 20 avril 2019. URL : http://journals.openedition.org/insitu/14639 ; DOI : 10.4000/insitu.14639

Ce document a été généré automatiquement le 20 avril 2019.

\section{(i) $\odot$

In Situ Revues des patrimoines est mis à disposition selon les termes de la licence Creative Commons Attribution - Pas d'Utilisation Commerciale - Pas de Modification 4.0 International. 


\section{Des archives de Jean-Claude Dondel aux archives de l'administration : des sources qui renseignent sur les collaborations entre architectes et artistes}

The archives of Jean-Claude Dondel and the archives of a public administration, sources illustrating the question of the collaboration between architects and artists

Anaïs Carré

1 Au sortir de la Seconde Guerre mondiale, après avoir donné la priorité à la reconstruction de l'appareil productif, l'État transfère son attention vers les logements et les bâtiments publics ${ }^{1}$, notamment les équipements scolaires: "À la nécessité de construire dans le domaine de l'habitat, correspond, dans quelque pays que ce soit, une autre nécessité : celle d'édifier les bâtiments scolaires utiles à la population jeune. Les deux problèmes sont conjoints " $\|^{2}$. L'État se dote d'une direction de l'Équipement scolaire universitaire et sportive (DESUS), placée sous l'autorité du ministère de l'Éducation nationale et ayant pour vocation de réaliser les établissements destinés à accueillir les élèves de tous niveaux. Parmi les nombreux architectes qui reçoivent l'agrément de la DESUS et se voient confier la réalisation des nouveaux établissements figure Jean-Claude Dondel (1904-1989), architecte ayant orienté sa carrière principalement vers les commandes publiques.

2 Après une formation académique à l'École des beaux-arts de Paris (atelier Roger-Henri Expert), Jean-Claude Dondel voit sa carrière personnelle prendre son essor à la suite de la construction du musée d'Art moderne de la Ville de Paris (actuel palais de Tokyo, 1935-1937, Jean-Claude Dondel, André Aubert, Paul Viard et Marcel Dastugue) destiné à recevoir les collections de la Ville de Paris et de l'État à l'occasion de l'Exposition 
internationale de 1937. Malgré la réception positive de cet édifice et la visibilité qu'elle donne à ses concepteurs, il faut attendre le début des années 1950 pour que Jean-Claude Dondel construise à nouveau des bâtiments publics. En 1955, lorsqu'il s'associe avec l'architecte Roger Dhuit ${ }^{3}$, Jean-Claude Dondel a déjà construit deux établissements scolaires. Cette association, ainsi que sa nomination en tant qu'architecte en chef des Bâtiments civils et palais nationaux en 1956, permettent à Jean-Claude Dondel (en association ou non avec Roger Dhuit) d'accéder à un nombre considérable de commandes pour des bâtiments scolaires. En effet, au cours de leurs onze années de collaboration, pas moins de trente établissements ont été construits sur l'ensemble du territoire français (annexe 1). Jean-Claude Dondel a, en outre, restauré le lycée Carnot (1 $17^{\mathrm{e}}$ arrondissement, Paris) et a participé au concours " conception construction " lancé par le ministère de l'Éducation nationale en 1962 afin de faire émerger des modèles d'établissements du second degré (collèges - lycées). Si l'architecte, reconnu pour ses qualités de praticien, accorde une place importante à la création d'architectures adaptées à l'enseignement et à la pédagogie, il met également un point d'honneur à faire participer les artistes à ses projets.

3 De façon évidente, le contexte s'y prête puisque l'arrêté du 18 mai 1951 prévoit que « les projets de constructions scolaires et universitaires devraient comporter un ensemble de

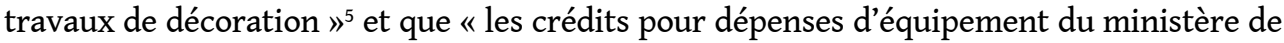
l'Éducation nationale, affectés à des travaux de décoration s'élèveraient au minimum à $1 \%$ du coût de la construction $»^{6}$. À partir de cette circulaire, les objectifs de la mesure du $1 \%$ artistique sont officiellement fixés : permettre à la jeune génération d'être en contact avec l'art contemporain, donner une certaine visibilité aux artistes et combattre l'aspect monotone dont souffrent certains bâtiments standardisés en les enrichissant d'œuvres d'art. Si l'architecte n'a aucun mérite personnel à associer les artistes à ses projets, la manière dont il envisage cette collaboration mérite d'être précisée. En effet, c'est dans l'optique de voir l'art faire « partie intégrante de l'architecture ${ }^{7}$ » que Jean-Claude Dondel sollicite les artistes dès la genèse du projet architectural. Afin d'examiner les modalités de ces collaborations, d'analyser les interactions entre l'architecture et l'art et d'évoquer l'histoire des œuvres d'art depuis leur création, diverses sources, parmi lesquelles les archives, peuvent être exploitées.

\section{Les fonds d'archives renseignant une production artistique réalisée au titre du $1 \%$}

4 À l'origine des établissements scolaires de Jean-Claude Dondel se trouvent en effet des commandes émanant du ministère de l'Éducation nationale. En tant qu'administration centrale de l'État, le ministère a obligation de verser ses archives aux Archives nationales ${ }^{8}$ . En ce qui concerne les documents relatifs à la construction des établissements scolaires, les ensembles documentaires sont classés en sous-séries en fonction des bureaux qui les produisent ${ }^{9}$. L'ensemble de ces éléments est conservé dans la série F/17 des Archives nationales ${ }^{10}$. Essentiellement composés de documents écrits, concernant la totalité des projets exécutés par le ministère de l'Éducation nationale, ces dossiers permettent de mieux appréhender l'histoire des commandes d'un point de vue administratif. Parmi ces documents, certains renseignent spécifiquement le $1 \%$ décoratif $^{11}$ et les dossiers des Beaux-Arts qui valident les projets. 
5 Si leur consultation est indispensable pour connaittre les tenants et les aboutissants des œuvres réalisées au titre $\mathrm{du} 1 \%$ décoratif au sein des établissements scolaires, le chercheur peut être frustré par le peu d'éléments d'informations que ces dossiers délivrent. En effet, ils permettent de connaître la nature de la commande et peuvent renseigner sur le choix des artistes ${ }^{12}$. Néanmoins, ils ne concernent que les aspects du projet dans lesquels intervient l'administration. Par conséquent, il est possible de mener une enquête comparative entre plusieurs dossiers d'édifices afin de déterminer si le cadre des collaborations est invariable ou si l'implication de certaines personnalités est susceptible de le faire évoluer. Une telle étude révèle que Jean-Claude Dondel adopte une démarche sensiblement différente de celle de ses collègues dans la mesure où il choisit personnellement les artistes avec lesquels il souhaite travailler. Elle révèle aussi que l'architecte procède toujours de la même manière. Cela ressort particulièrement des archives qui émanent de la sous-direction de la Création architecturale et des Constructions publiques, organe qui dépend de la direction de l'Équipement scolaire, universitaire et sportif ${ }^{13}$.

6 Les archives de Jean-Claude Dondel, dont l'architecte a lui-même fait don à la direction des Archives de France (DAF) ${ }^{14}$, apportent un point de vue complémentaire sur ses collaborations avec les artistes. Déposé au Centre d'archives de l'Institut français d'architecture (IFA) en février $1989^{15}$, soit quelques mois avant le décès de l'architecte, le fonds couvre toute la période d'activité de Jean-Claude Dondel, de 1935 jusqu'à 1977, incluant ses années d'association avec Roger Dhuit. La grande majorité des projets et des réalisations de Jean-Claude Dondel y sont documentés par des écrits, de la correspondance, des photographies et, naturellement, des plans. Divisé en sous-fonds classés dans l'ordre alphabétique (A, B, C, D), le fonds Jean-Claude Dondel répond à une logique thématique et chronologique. Le premier sous-fonds, composé des "pièces personnelles, professionnelles et documentation », permet d'appréhender la personnalité de l'architecte par le biais des correspondances d'agence et des courriers personnels. Quelques lettres font ainsi état des échanges de Jean-Claude Dondel avec les artistes, à l'exemple de celles échangées avec Laszlo Barta ${ }^{16}$. Le second sous-fonds concerne les établissements scolaires. Il témoigne des lacunes qui peuvent exister au sein d'un fonds d'archives puisque même si l'ensemble des documents est particulièrement abondant - le fonds est composé de quatre-vingt-seize boîtes d'archives cotées 074 IFA de 1 à 96 , de trente-sept boîtes à rouleaux cotées de 100 à 136 - il ne documente pas l'ensemble de la production de l'architecte dans ce domaine. Certains bâtiments, comme le lycée MauriceRavel (20 arrondissement, Paris, 1955-1962), n'y figurent pas.

$7 \mathrm{Au}$ Centre d'archives d'architecture $\mathrm{du} \mathrm{xx}^{\mathrm{e}}$ siècle, un second fonds s'avère primordial pour appréhender la production architecturale de Jean-Claude Dondel et de ses confrères ayant œuvré au $\mathrm{Xx}^{\mathrm{e}}$ siècle $^{17}$. Ce fonds est celui de la direction de l'Architecture et de l'Urbanisme (DAU) ${ }^{18}$. Comme le fonds de la DESUS ou celui des Beaux-Arts, le fonds de la DAU témoigne d'une activité administrative particulière. En effet, il est essentiellement composé de dossiers de références réunissant des curriculum vitae d'architectes, des listes de réalisations et des documents présentant ces dernières, recueillis directement par la DAU auprès des architectes ou de leurs agences ${ }^{19}$. Le dossier de Jean-Claude Dondel se compose de son curriculum vitae et de plusieurs fiches relatives à ses réalisations, documents qui témoignent d'une activité en cours puisque l'architecte les a transmis en avril 1967. Pour chacun des établissements scolaires qu'il choisit de présenter, l'architecte détaille le nom des artistes qui ont collaboré au projet et le titre des œuvres réalisées, ce 
qui n'est pas le cas par exemple de son associé Roger Dhuit ${ }^{20}$. Il accompagne d'ailleurs la notice écrite par quelques photographies donnant à voir l'œuvre d'art, plutôt que l'architecture. C'est le cas, par exemple, du lycée technique de Bourges. L'établissement comporte un pavillon administratif, un externat, un internat et un atelier avec gymnase incorporé. Pour le présenter, l'architecte constitue un dossier comprenant une vue des bâtiments depuis la cour de récréation, une vue de l'internat ainsi que des clichés de deux des trois œuvres réalisées dans le cadre du $1 \%$ décoratif ${ }^{21}$. Ces photographies (fig. 1) permettent de découvrir le détail du panneau décoratif en mosaïque réalisé par Georges Rohner ainsi qu'une statue du sculpteur Marcel Gili. Les deux œuvres font écho à l'enseignement dispensé dans l'établissement. En effet, la mosaïque illustre le travail du métal que l'artiste évoque en représentant la manipulation d'un marteau et d'une pince au-dessus d'une vasque contenant des flammes tandis qu'à gauche sont figurés les tubes métalliques ainsi fabriqués. La sculpture de Marcel Gili représente un homme en mouvement (l'un de ses genoux est plié) caché derrière une sculpture monumentale qu'il étreint. L'homme et l'élément qu'il enlace semblent indissociables tant et si bien qu'il n'est pas possible de déterminer si l'homme est en train de réaliser cette sculpture, de la poser ou encore de la déplacer. Il n'empêche que l'œuvre renvoie à l'acte même de création dans le but d'interpeller les élèves sur leur propre pouvoir d'invention. Parmi les autres photographies d'œuvres d'art présentes dans le dossier DAU de Jean-Claude Dondel figurent les "écrans en dalles de verre de couleur [des] préaux ", réalisés par André Pierre pour l'ensemble scolaire d'Annonay (Ardèche, 1963-1965) et la mosaïque de Laszlo Barta habille la façade d'entrée du gymnase du lycée Édouard-Herriot de Voiron (Isère, 1954-1963). Force est de constater que Jean-Claude Dondel choisit de présenter en priorité les œuvres installées en extérieur, bien que des mosaïques de Laszlo Barta ornent aussi les internats de la cité scolaire d'Annonay. Il est aussi important de préciser que Jean-Claude Dondel légende ces clichés en précisant l'emplacement des œuvres dans l'établissement, mais sans indiquer systématiquement ni le nom de l'artiste ni le titre de l'œuvre. Pour l'architecte, l'œuvre reste indissociable du projet architectural, tout en lui étant subordonnée. 


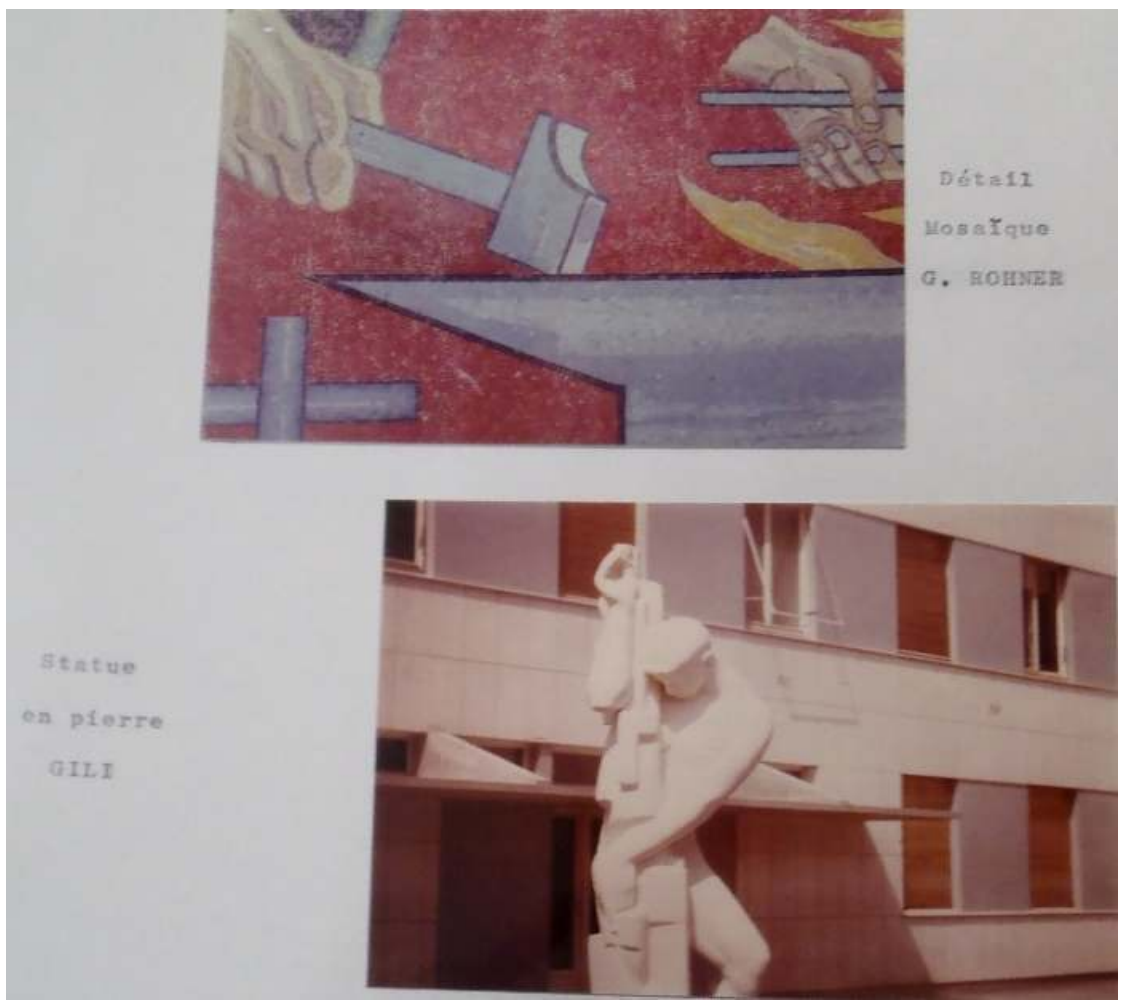

Lycée de Bourges (Cher), s. d., arch. : Jean-Claude Dondel et Roger Dhuit : détails des œuvres de Marcel Gili et Georges Rohner, s. d., CAA du Xxe siècle, fonds DAU, Jean-Claude Dondel, 133 Ifa 84/05.

Phot. Anaïs Carré. (c) Anaïs Carré.

Si les archives de l'architecte et de l'administration sont nécessaires pour comprendre le cadre de la collaboration, les archives des artistes constituent également une précieuse source d'informations. Malheureusement, dans le cas de Jean-Claude Dondel, il n'existe aucun fonds de plasticien qui puisse fournir de contrepoint aux archives de l'architecte. En dehors du fonds Jean-Claude Dondel et des fonds précédemment cités, d'autres sources renseignent le chercheur sur ces collaborations. En effet, le fonds des Beaux-Arts conservé aux Archives nationales permet de tracer les contours de ces commandes publiques. Les versements concernant les commandes et les achats d'œuvres d'art par l'État au cours $\mathrm{du} \mathrm{xx}^{\mathrm{e}}$ siècle sont classés dans un sous-fonds ${ }^{22}$. Les achats sont présentés par dossiers, classés selon l'ordre alphabétique des noms des artistes. Ainsi, lorsque l'identité d'un artiste ayant participé à la réalisation d'une œuvre d'art dans un établissement scolaire construit par Jean-Claude Dondel est déjà connue du chercheur, la consultation de ces archives permet de recenser l'ensemble des commandes que l'artiste a déjà reçu, documentant par là-même les collaborations de ce dernier avec d'autres architectes. Au fil des dossiers, il apparaît sans surprise que Jean-Claude Dondel ne fait pas appel à des inconnus et que chaque artiste sollicité a déjà, au moins une fois, reçu une commande de l'État ${ }^{23}$. On peut légitimement penser qu'en prenant cette précaution, JeanClaude Dondel s'assure du succès de ces choix: lorsque les œuvres passent devant la commission des achats de l'État, leurs auteurs sont déjà connus de l'administration, ce qui favorise leur agrément. 


\section{L'affirmation d'une volonté personnelle}

9 Les archives se révèlent donc être des sources particulièrement importantes pour analyser la démarche de Jean-Claude Dondel. Parmi les autres types de documents renseignant significativement la place que l'architecte accorde à l'art et la manière dont il fait interagir les œuvres d'art avec l'architecture figurent les sources imprimées. Une interview de Jean-Claude Dondel réalisée par la journaliste Henriette Psichari pour la revue hebdomadaire d'information pédagogique L'Éducation nationale, en 1966, invite par exemple l'architecte à s'exprimer sur la place de l'art dans la construction scolaire. Dans l'article, Jean-Claude Dondel est présenté comme l'auteur de «beaucoup de lycées de Paris et de groupes scolaires [...] également en banlieue et même en province ${ }^{24} »$ et, par conséquent, comme un architecte de référence en matière d'architecture scolaire. Il y affirme que «le $1 \%$ fait partie intégrante de l'architecture. Quand [il fait] un plan, [il] incorpore dans son projet ici une statue, là une tapisserie, ailleurs un mur coloré ${ }^{25}$. Cette affirmation mérite d'être complétée par l'étude des documents qui constituent le projet d'architecture : plan, esquisses, devis, et. Dans le cas présent, les propos de Jean-Claude Dondel trouvent confirmation dans les documents conservés au sein de son fonds d'archives ${ }^{26}$. En effet, pour exemple, les premiers plans du lycée Gabriel-Fauré (Paris, $13^{\mathrm{e}}$ arrondissement, 1959-1964) présentent "un motif décoratif ${ }^{27}$ » sur la façade est du gymnase et, à l'entrée de l'établissement, une sculpture représentant deux personnages attablés. Ces œuvres ont spécifiquement été pensées pour l'emplacement qu'avait choisi l'architecte. Ainsi, lorsque le projet est soumis à la commission de la DESUS, les œuvres d'art font déjà partie intégrante du programme, donc des éléments à réaliser. Les artistes qui participent à ce projet sont l'artiste-peintre Georges Rohner et le sculpteur Olivier Descamps. Par arrêté du 9 mai 1961, Georges Rohner est officiellement chargé de réaliser une grande peinture murale (fig. 2), de 10,85 x 5,05 mètres environ, décrite comme « une composition à partir d'éléments géométriques " pour 32000 nouveaux francs; quant à Olivier Descamps, il reçoit 38000 nouveaux francs pour une sculpture en bronze coulé et soudé, montée sur socle en cuivre (2,50 x 3,50 x 1,20 mètres), représentant L'Astrologie et la Biologie (fig. 3), conformément à la maquette acceptée par l'administration. D'une manière générale, Jean-Claude Dondel « indique même l'artiste qu'il souhaite, bien que ce soit [toujours] la commission des achats de l'État qui décide $»^{28}$. Par conséquent, il fait appel aux artistes dès qu'il reçoit commande pour la réalisation d'un établissement afin de pouvoir travailler avec eux en amont du projet, alors que, d'une manière générale, les architectes se voient proposer des artistes par le secrétariat des Beaux-Arts, cela bien après avoir établi les plans pour la construction du bâtiment ${ }^{29}$. 
Figure 2

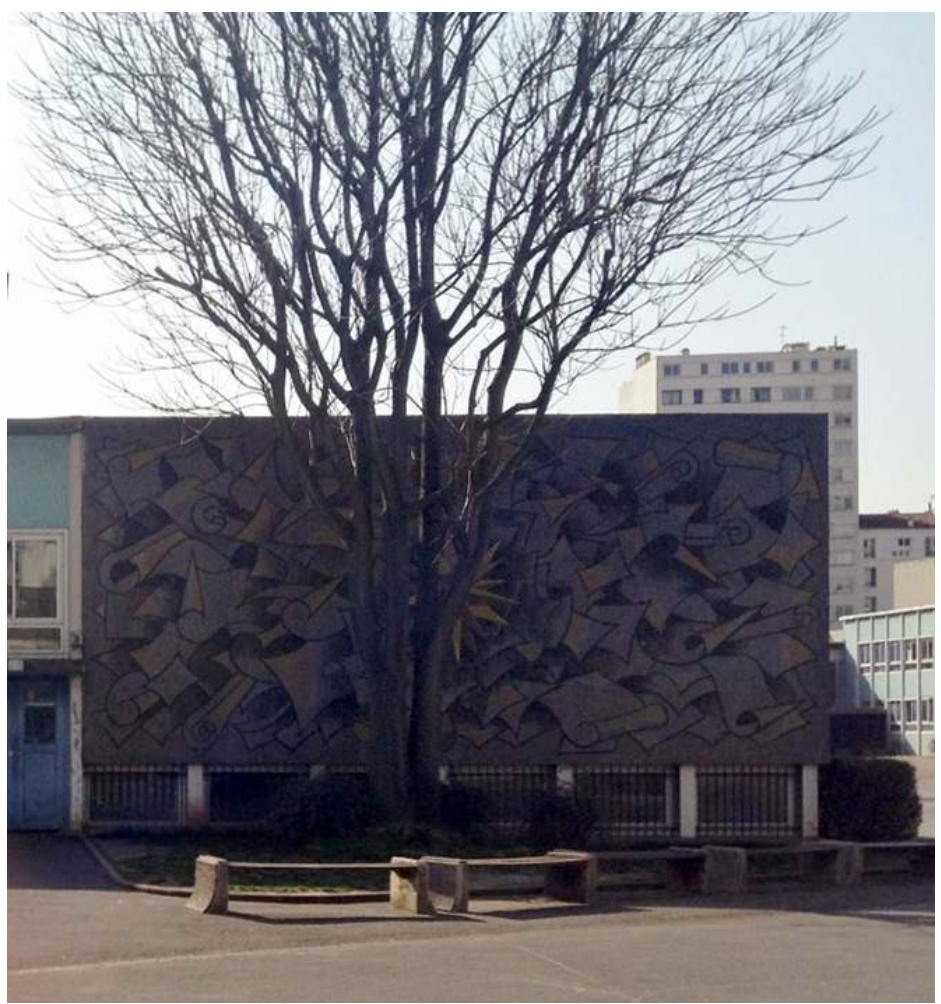

Lycée Gabriel-Fauré (Paris, 13e arrondissement), 1957-1964, arch. Jean-Claude Dondel et Roger Dhuit : œuvre de Georges Rohner, mosaïque en pâte de verre.

Phot. Anaïs Carré, avril 2015. ㄷ Anaïs Carré. 
Figure 3

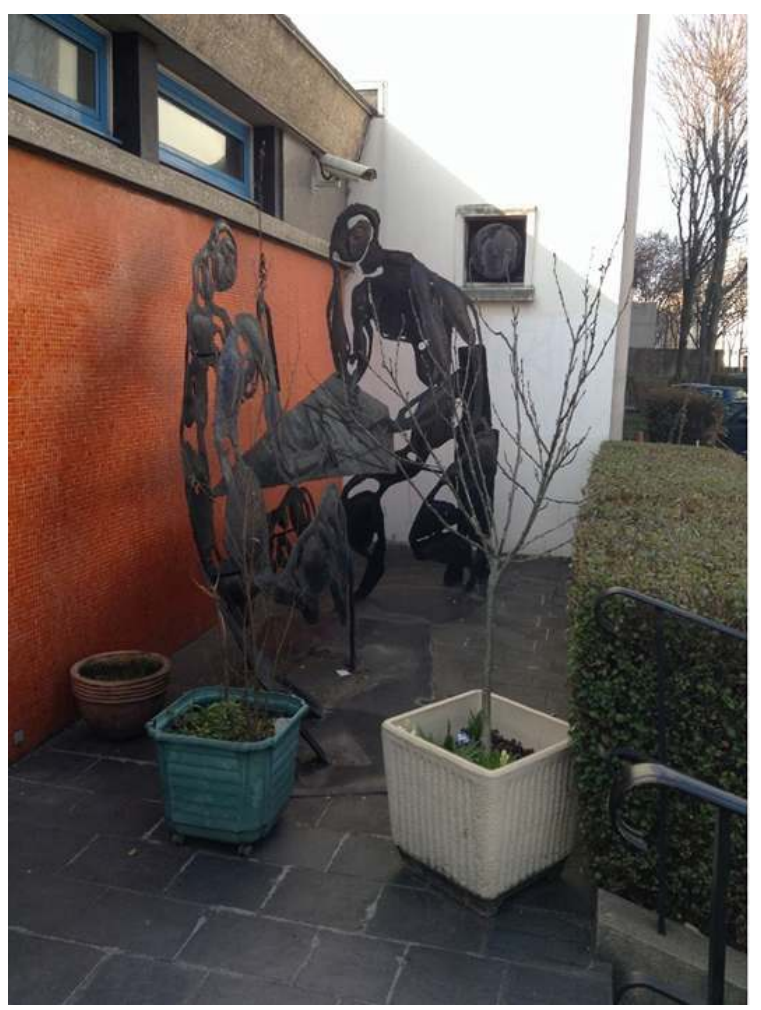

Lycée Gabriel-Fauré (Paris, 13e arrondissement), 1957-1964, arch. Jean-Claude Dondel et Roger Dhuit : œuvre d'Olivier Descamp située à l'entrée de l'établissement.

Phot. Anaïs Carré, avril 2015. (c) Anaïs Carré.

\section{Des œuvres à l'épreuve du temps : le cas du lycée Paul-Valéry}

Parmi les réalisations de Jean-Claude Dondel à Paris, le lycée Paul-Valéry est le plus richement doté en œuvres d'art. En 1966, le proviseur du lycée Paul-Valéry, M. Marchal s'exprime sur le fait que « l'idée n'était pas de planter n'importe quelle œuvre d'art sur n'importe quel mur, mais de créer une atmosphère d'art et de liberté $»^{30}$, ce qui témoigne d'un accueil positif de la démarche de l'architecte. Mais certaines œuvres ont vu leur destin contrarié par une réception moins enthousiaste et par les méfaits du temps. Le groupe sculpté La Famille, réalisé par Robert Couturier en 1960 pour le même lycée, a été retiré peu après l'ouverture de l'établissement. Ce groupe, représentant un couple avec un jeune enfant dans les bras de sa mère, était placé au centre d'un parterre végétalisé situé à l'entrée du lycée. Plus haut que le bâtiment des salles de classe, ce bronze était visible depuis la rue et ne fut pas apprécié de tous. Un article de L'Auto-journal ${ }^{31}$, après l'avoir qualifié ironiquement de " chef-d'œuvre », insiste sur ce " loupé » et demande aux autorités de "reprendre » et de "soustraire à la vue du public » cette représentation «triste » de la famille. Rien n'indique que cet article ait influencé les parents d'élèves du lycée, mais ceux-ci signent une pétition à l'encontre de la sculpture qui est finalement déposée. Jean-Claude Dondel ne peut qu'exprimer ses regrets face au retrait de l'œuvre de Couturier, d'autant que l'architecte entendait « œuvrer » pour les élèves plus que pour 
l'État lorsqu'il concevait un établissement ${ }^{32}$, et qu'en l'occurrence, cette œuvre était appréciée par les élèves, opinion confirmée par Sylvie Chio, qui fut élève dans l'établissement de 1959 à $1966^{33}$. La Famille de Couturier n'est pas la seule œuvre du lycée Paul-Valéry qui ait disparu. Il y a quelques années, le mural en pavés de verre de Max Ingrand (fig. 4) qui séparait les deux cours de l'établissement sous l'un des deux préaux a été démonté pour des raisons de sécurité. Décrite à sa livraison comme une «large bande de vitraux modernes, des plaques de verre de toutes couleurs enchâssés dans du ciment noir. Le soleil levant les transperce le matin et le couchant les illumine de l'autre côté l'après-midi. Filles et garçons ne se lassent pas de suivre les rayons du soleil parisien jouant avec la couleur $~^{34}$, l'œuvre était composée de pavés colorés enchâssés dans du béton ${ }^{35}$. Les deux panneaux réalisés pour la cité scolaire d'Annonay ${ }^{36}$ par André Pierre ont connu le même sort ${ }^{37}$.

\section{Figure 4}

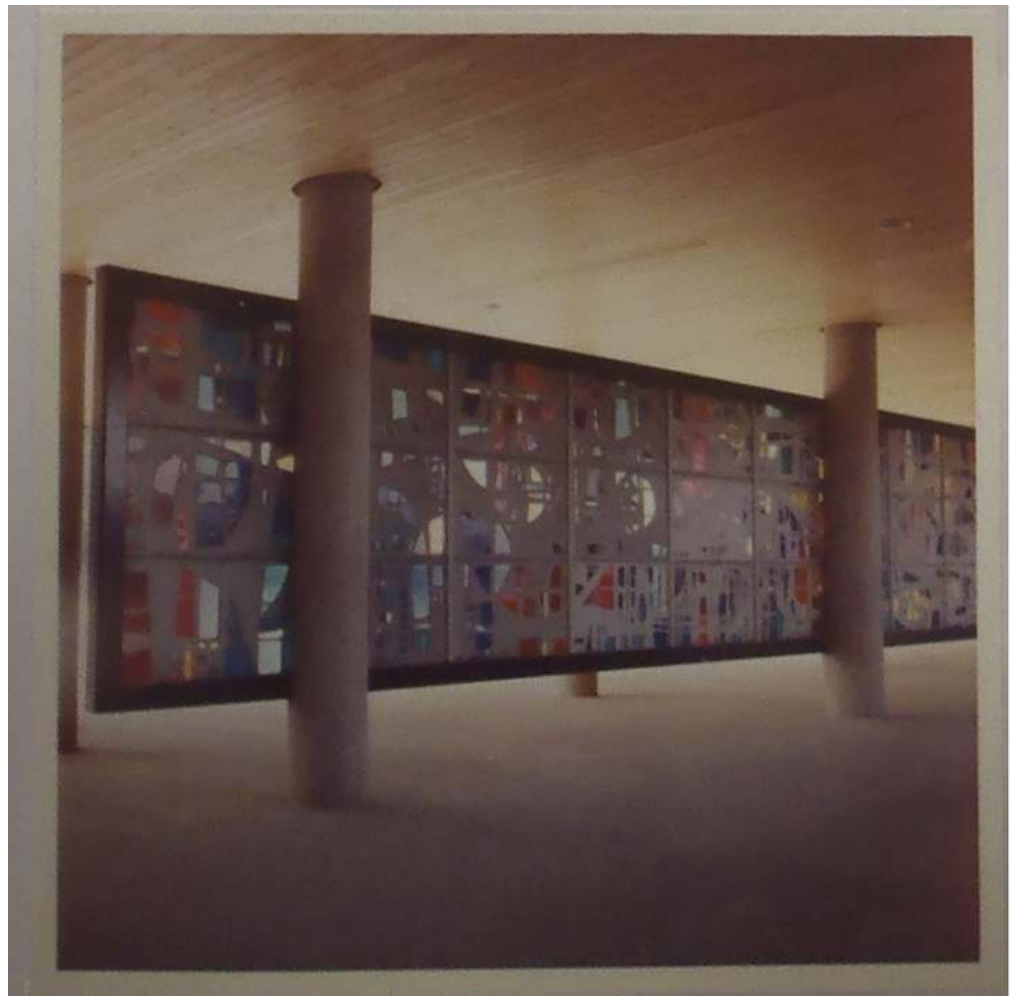

Lycée Paul-Valéry (Paris, 12e arrondissement), 1958-1963, arch. Jean-Claude Dondel et Roger Dhuit : photographie de l'écran de verres colorés de Max Ingrand, (s. d. circa 1958, CAA du xxe siècle, DAU 378 IFA 84/050).

Phot. Anaïs Carré. (c) Anaïs Carré.

11 Aujourd'hui, seules deux œuvres sont conservées et demeurent à leur place d'origine dans le lycée Paul-Valéry : la grande tapisserie du hall d'entrée du petit pavillon administratif et le jardin japonais ${ }^{38}$ situé à l'arrière de ce pavillon. La tapisserie de Pierre Despierre représente Prométhée, nu, vu de dos et entouré de deux éclairs rouges (fig. 5). L'artiste l'a figuré au centre d'un monde bipartite : l'un grouillant d'animaux marins et l'autre de végétaux. Dans cette œuvre, les contrastes colorés jouent un rôle important puisque l'homme entièrement blanc se détache d'un fond coloré. Bien que toujours présentes, ces œuvres sont menacées par une restructuration de l'établissement ${ }^{39}$ programmée en 2017 par la région et la Ville de Paris et qui prévoit de détruire les bâtiments des classes et de 
l'administration, ainsi que le jardin japonais situé entre les deux. Au mieux, il ne restera donc de l'ambitieux projet de Jean-Claude Dondel pour le lycée Paul-Valéry que la tapisserie de Jacques Despierre. Cette œuvre imaginée pour faire partie intégrante de l'architecture va se retrouver détachée du bâtiment pour lequel elle avait été créée. Par conséquent, même si elle trouve place dans le nouvel établissement, la tapisserie ne répondra plus à la conception que Jean-Claude Dondel avait des liens étroits que doivent entretenir l'art et l'architecture. Les archives seront alors les seuls témoins de cette conviction.

\section{Figure 5}

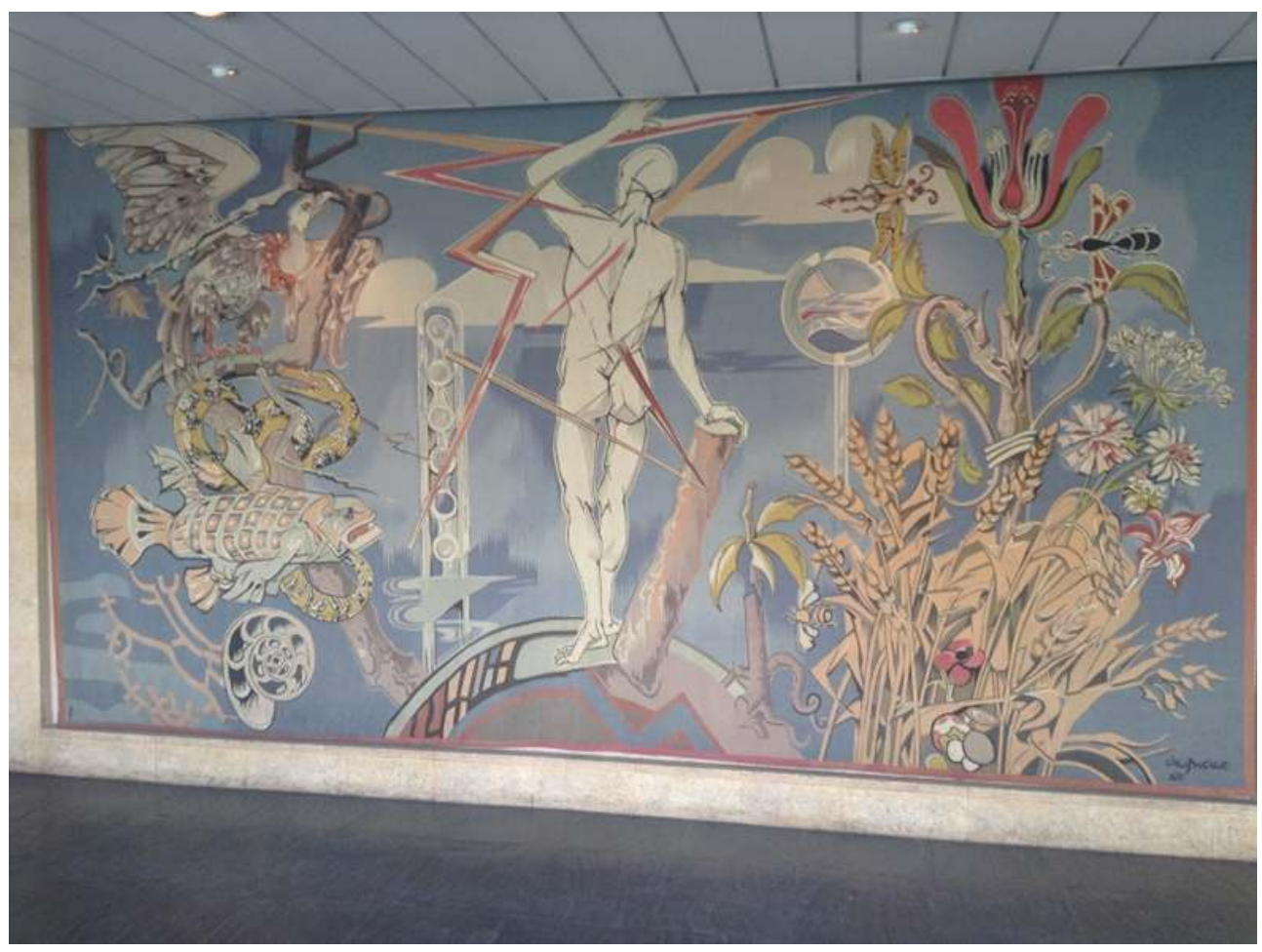

Lycée Paul-Valéry (Paris, 20 arrondissement), 1958-1963, arch. Jean-Claude Dondel et Roger Dhuit : vue actuelle de la tapisserie de Pierre Despierre dans le pavillon administratif.

Phot. Carré, Anaïs, avril 2015. (c) Anaïs Carré.

En 1950, la revue L'Éducation nationale proposait un numéro spécial "Architecture mobilier et décoration scolaires » dans lequel la directrice du Centre international de pédagogie, Edmée Hattinguais, signait un article sur « la décoration des établissements du second degré $»^{40}$. Elle y présentait l'intérêt de la présence d'œuvres d'art dans les lycées :

En s'écartant des poncifs et d'une certaine mièvrerie, les artistes s'efforceront de traiter de grands thèmes d'une beauté éternelle, sans concession complaisante à leur public, sans effort inutile d'adaptation. Si leurs œuvres sont belles, elles seront par là même éducatrices, mais les commissions chargées du choix devront prendre de grandes précautions, car l'œuvre médiocre se démode rapidement et, si une statue peut à la rigueur être déplacée, une fresque s'impose et risque de devenir ridicule ${ }^{41}$.

13 Jean-Claude Dondel partage les mêmes convictions et aspire à offrir un espace favorable au développement des élèves, dans lesquels l'unité entre les arts et l'architecture fasse sens. À l'heure où certains de ces bâtiments ont été détruits ${ }^{42}$ - ou sont en passe de l'être ${ }^{43}$ - et où certaines œuvres d'art ont disparu, les archives permettent encore d'établir une 
analyse plus ou moins précise des bâtiments et de leurs programmes décoratifs. Leur consultation et leur valorisation, par le biais d'expositions ou de publications, devraient en outre être mises à profit pour mieux appréhender l'avenir de bâtiments dans lesquels architecture et œuvres d'art furent indissociablement liées.

\section{Annexe : Liste des bâtiments scolaires construits par Jean-Claude Dondel et Roger Dhuit}

Les bâtiments scolaires construits par Jean-Claude Dondel et Roger Dhuit vont de l'école maternelle à l'université en passant par les cités scolaires, les collèges d'enseignement secondaires, généraux ou techniques et les lycées. Ont été recensés les établissements suivants :

centre d'apprentissage de Gelos (Pyrénées-Atlantiques, 1953) ;

lycée Édouard-Herriot (Voiron, Isère, 1954-1963) ;

lycée Maurice Ravel (Cours de Vincennes, Paris 20e 1955-1962) ;

école maternelle Carnot (Sannois, Val-d'Oise, 1957-1967) ;

lycée Paul-Valéry (boulevard Soult, Paris $12^{\mathrm{e}}$, 1958-1963);

lycée Gabriel-Fauré (avenue de Choisy, Paris 13e, 1959-1964);

centre d'apprentissage masculin du bâtiment (Brest-Le Bouguen, Finistère, 1959-1965) ;

école normale supérieure de jeunes filles (Montrouge, Hauts-de-Seine, 1963);

cité scolaire (Annonay, Ardèche, 1963-1965) ;

collège d'enseignement secondaire (Drancy, Seine-Saint-Denis, 1967-1969) ;

collège d'enseignement secondaire (Loches, Indre-et-Loire, 1967-1969) ;

collège d'enseignement secondaire (Riom, Puy-de-Dôme, 1967-1969) ;

collège d'enseignement secondaire (Les Clayes-sous-Bois, Yvelines, 1968-1969) ;

collège d'enseignement général (Rosières-en-Santerre, Somme, 1968-1969) ;

collège d'enseignement général (Genlis, Côte-d'Or, 1968-1970) ;

cité scolaire (Charleville-Mézières, Ardennes 1969) ;

collège d'enseignement général (Levroux, Indre, 1969-1967) ;

faculté de Lettres et de Sciences humaines (Metz, Moselle, 1970) ;

collège d'enseignement secondaire (Montrouge, Hauts-de-Seine, 1970) ;

collège d'enseignement secondaire (Montfort-l'Amaury, Yvelines, 1970-1973) ;

collège d'enseignement secondaire (Lempdes, Puy-de-Dôme, 1970-1974) ;

collège d'enseignement secondaire (Chelles, Seine-et-Marne, 1970-1975) ;

cité scolaire (Évry, Essonne, 1970-1975) ;

collège d'enseignement secondaire (Oullins, Rhône, 1971-1972);

groupe primaire La Fleurie (Lempdes, Puy-de-Dôme, 1971-1973) ;

collège d'enseignement secondaire (Gap, Hautes-Alpes, 1971-1974) ;

collège d'enseignement secondaire (Bourg-lès-Valence, Drôme, 1971-1975) ;

collège d'enseignement secondaire (Lyon, Rhône, 1971-1975) ; 


\section{NOTES}

1. - VOLDMAN, Danièle. La reconstruction des villes françaises de 1940 à 1954, histoire d'une politique. Paris : L'Harmattan, 1997.

2. - Ibid.

3. - Architecte des Bâtiments civils et Palais nationaux, agréé par le ministère de la Reconstruction et de l'Urbanisme pour les départements de la Seine, de la Seine-et-Oise et du Calvados.

4. - L'ensemble des projets du concours est conservé aux Archives nationales, fonds du ministère de l'Éducation nationale, sous-fonds de la DESUS, 19780671/32 F17 bis 20622. La revue Techniques et architecture a publié en janvier 1964 un numéro spécial sur le concours «conceptionconstruction » du ministère de l'Éducation nationale documentant l'ensemble de ce dernier.

5. - Arrêté du 18 mai 1951 d'utilisation des crédits pour des travaux de décoration dans les établissements d'enseignement, Journal officiel de la République française, 17 juin 1951, p. 6370.

6. - Ibid.

7. - PSICHARI, Henriette. «L'Art vivant dans les lycées ». L'Éducation Nationale, 1966, s. p.

8. - Articles L. 212-13 et L. 212-14 du Code du patrimoine.

9. - Par exemple les sous-séries émises par la direction des Équipements et des constructions, la sous-direction de la Création architecturale et des Constructions publiques, division des Constructions scolaires et du conseil aux collectivités (ministère de l'Éducation nationale), la sous-direction des affaires générales et financières (direction des Équipements et des Constructions, ministère de l'Éducation nationale), le service des Cartes universitaire et scolaire et du plan d'équipement (ministère de l'Éducation nationale) et la division des Constructions scolaires et du conseil aux collectivités (ministère de l'Éducation nationale).

10. - Voir le site des Archives nationales, État général des fonds des Archives nationales, mis à jour en 2009: http://www.archivesnationales.culture.gouv.fr/chan/chan/fonds/F17_2009.pdf [consulté le 12/12/2015].

11. - Archives nationales, fonds du ministère de l'Éducation nationale, direction de l'Équipement scolaire, universitaire et sportif, sous-direction de la Création architecturale et des constructions publiques, 19810663/1-1156.

12. - Cela n'est malheureusement pas toujours le cas, notamment en ce qui concerne les bâtiments de Jean-Claude Dondel.

13. - AN, site de Pierrefitte, fonds du ministère de l'Éducation nationale, sous-direction de la création architecturale et des constructions publiques, 19810663/731, 19810663/735, $19810663 / 378,19810663 / 880$.

14. - Service patrimonial du ministère de la Culture et de la Communication, la direction des Archives de France, créée en 1897, est devenue en 2009 le Service interministériel des Archives de France (SIAF).

15. - Voir le site du Centre d'archives d'architecture du $\mathrm{xx}^{\mathrm{e}}$ siècle, inventaire en ligne du fonds Jean-Claude Dondel: http://archiwebture.citechaillot.fr/fonds/FRAPN02_DONJE/inventaire [consulté le 12/12/2015]. 
16. - Fonds Jean-Claude Dondel, 074 IFA Donje-A-01, caisses 1 à 6 et 94, Centre d'archives d'architecture du xx $x^{\mathrm{e}}$ siècle.

17. - Les archives de la direction de l'Architecture et de l'Urbanisme s'avèrent par conséquent être une source primordiale pour le chercheur. Il n'en demeure pas moins qu'elles sont lacunaires puisqu'une partie des documents émis par le ministère de l'Équipement n'est pas encore communicable au public. Ainsi, pour exemple, si le dossier 378 IFA 84/5 permet de visualiser de nombreuses photographies d'œuvres d'art réalisées - telles que le panneau décoratif en mosaïque de Georges Rohner et la statue de Marcel Gili pour le lycée de Bourges, «l'écran en dalle de verres colorés» par André Pierre pour la cité scolaire d'Annonay, la mosaïque décorative de Laszlo Barta pour le lycée Édouard-Herriot de Voiron, un des deux panneaux « en dalle de verres colorés » par Max Ingrand au lycée Paul-Valéry, la peinture murale de Georges Rohner et la sculpture en bronze d'olivier Descamps pour le lycée Gabriel-Fauré, le dossier 133 IFA 503/49 est encore soumis à un délai de communication. En effet, bien qu'il n'y ait pas de restriction juridique à la consultation du fonds, les boîtes 133 IFA 500 à 511, contenant des jugements sur des réalisations architecturales, ne sont pas communicables pendant 50 ans (Code du patrimoine, art. L 213.2 \$I $3^{\circ}$ ).

18. - Voir le site du Centre d'archives d'architecture du $x^{e}$ siècle, inventaire en ligne du fonds de la direction de l'Architecture et de l'Urbanisme: http://archiwebture.citechaillot.fr/fonds/ FRAPN02_DAU [consulté le 12/12/2015]. Comme l'indique l'instrument de recherche élaboré par le Centre d'archives d'architecture, ce fonds "représente une coupe significative sur l'architecture française de l'époque, puisqu'il illustre les travaux de presque 1800 agences sur les quelque 8000 que comptait la France vers 1970 ».

19. - Ibid.

20. - Voir le site du Centre d'archives d'architecture du $x^{e}$ siècle, inventaire en ligne du fonds de la direction de l'Architecture et de l'Urbanisme, dossier de Roger Dhuit, 133 Ifa 82/2 : http:// archiwebture.citechaillot.fr/fonds/FRAPN02_DAU/inventaire [consulté le 12/12/2015].

21. - La fiche de présentation de l'établissement dans le dossier DAU de Jean-Claude Dondel fait état «d'un panneau décoratif en mosaïque » par Georges Rohner, d'une statue réalisée par Marcel Gili et d'une fresque de Brayer.

22. - Voir le site des Archives nationales, commandes et achats d'œuvres d'art par l'État ( $\mathrm{xx}^{\mathrm{e}}$ siècle) : https://www.siv.archives-nationales.culture.gouv.fr/siv/IR/FRAN_IR_000227 [consulté le 12/12/2015].

23. - Aux Archives nationales, dans la section Beaux-Arts, classés en F21, les dossiers de commande de Jacques Despierre sont conservés ( $\mathrm{F} / 21 / 6919)$; on dénombre vingt commandes et achats pour cet artiste entre 1951 et 1960 . Sous la cotation F/21/6951 sont consultables les dossiers de Laszlo Barta et Paul Belmondo; ceux des œuvres de Marcel Gili sont réunis en F/21/6925 et ceux de Georges Rohner en F/21/6942.

24. - PSICHARI, Henriette. Art. cit.

25. - Ibid.

26. - Se reporter au fonds Jean-Claude Dondel (Centre d'archives d'architecture du xx siècle, 074

IFA). L'ensemble des documents conservés dans le sous-fonds «B» sont consacrés aux établissements scolaires (074 IFA Donje-B-01 à 074 IFA Donje-B-30).

27. - Terme utilisé par l'architecte sur les plans conservés dans le fonds Dondel (074 IFA DonjeB-05).

28. - PSICHARI, Henriette. Art cit.

29. - AN, fonds du ministère de l'Éducation nationale, direction Équipement scolaire, universitaire et sportif, sous-direction de la Création architecturale et des Constructions publiques, 19810663/1-1156.

30. - PSICHARI, Henriette. Art. cit. 
31. - DELILLE, Richard. «La main droite... et la main gauche ». L'Auto-journal, octobre 1960, s. p. Richard Delille décrit le groupe père-mère-enfant comme " émouvant, un peu douloureux » en concluant que « la vie est lourde à ce couple de parents».

32. - Dans les dernières lignes de l'article d'Henriette Psichari, Jean-Claude Dondel précise ainsi que lorsque les œuvres d'art sont appréciées par les élèves, il considère qu'il a réussi ce pourquoi il a été engagé (PSICHARI, Henriette. Art. cit.).

33. - Le témoignage fut publié dans l'ouvrage Racontez-moi Paul-Valéry. Histoires et mémoire de la communauté scolaire. Paris : 2001, p. 40. Bien qu'anecdotique, il permet de confirmer les dires de l'architecte, mais également de les nuancer : «Pour ceux qui ne connaissent que l'aspect actuel, j'aimerai parler de la statue, la fameuse statue représentait un couple avec un enfant. La femme et l'enfant étaient habillés, l'homme était nu. Elle avait été exécutée, d'après la "rumeur ", d'après un dessin d'enfant. Moi, elle me plaisait bien cette statue, de style moderne, je trouvais qu'elle s'harmonisait bien avec le bâtiment. Malheureusement tout le monde n'était pas de cet avis et régulièrement des lycéens facétieux venaient la nuit pour peindre un slip blanc, pour habiller un peu l'homme qui devait avoir froid! Elle a été enlevée... et a séjourné longtemps dans les sous-sol du lycée ".

34. - PSICHARI, Henriette. Art. cit.

35. - N'ayant jamais été entretenus les vitraux se détachèrent les uns après les autres et les élèves considéraient le mural comme un élément vétuste et sans valeur. Afin d'éviter tout accident, l'administration décida de supprimer totalement le panneau malgré le mécontentement de la famille de Max Ingrand. Désormais, seuls les poteaux sur lesquels le grand panneau était fixé gardent les traces de sa présence.

36. - Comme nous pouvons le constater sur les photographies de l'établissement conservées dans le dossier Jean-Claude Dondel (fonds DAU, CAA du xx ${ }^{\mathrm{e}}$ siècle).

37. - En 2010, l'établissement a partiellement été détruit pour laisser place à de nouveaux locaux. Les murs de verre font partie des éléments supprimés à cette occasion. Par conséquent, les photographies conservées dans le dossier Jean-Claude Dondel (fonds de la DAU, CAA) sont des documents essentiels sur l'existence de ces éléments décoratifs.

38. - Dont l'auteur reste inconnu malgré la diversité des fonds d'archives et les sources imprimées documentant la construction du lycée Paul-Valéry.

39. - « La cité scolaire Paul-Valéry sera rasée et reconstruite ». Le Parisien, 15 février 2016. Voir sur le site: http://www.leparisien.fr/paris-75/paris-75012/paris-la-cite-scolaire-paul-valerysera-rasee-et-reconstruite-15-02-2016-5548439.php\#xtref=https\%3A\%2F\%2Fwww.google.fr [consulté le 15/02/2016].

40. - HATTINGUAIS, Edmée. «La décoration des établissements du second degré ». L'Éducation nationale, $\mathrm{n}^{\circ}$ spécial « Architecture mobilier et décoration scolaires », mai 1950, p. 44-45.

41. - Ibid.

42. - À l'exemple de la cité scolaire d'Annonay (Ardèche, 1963-1965) qui a fait l'objet d'une restructuration en 2010.

43. - En 2015, la région, qui a en charge l'entretien des établissements - depuis la décentralisation en 1983 - a annoncé un projet de reconstruction pour le lycée Paul-Valéry afin de séparer les deux cycles d'enseignement dispensés dans la cité scolaire. L'état du bâtiment des classes, jugé vétuste et inadapté "aux conditions actuelles d'enseignement", amène la région à vouloir le détruire pour laisser place à un nouvel établissement. 


\section{RÉSUMÉS}

Au sortir de la Seconde Guerre mondiale, l'État crée une administration placée sous l'autorité du ministère de l'Éducation nationale ayant pour vocation de réaliser les établissements scolaires devant accueillir les élèves de tous les niveaux, la direction de l'Équipement scolaire universitaire et sportif (DESUS). Entre 1950 et 1970, elle sollicitera de nombreux architectes, dont Jean-Claude Dondel (1904-1989) qui réalise de nombreux établissements sur l'ensemble du territoire français. Ses réalisations se caractérisent par la volonté de voir le $1 \%$ décoratif faire partie intégrante de l'architecture. Cet article propose d'évoquer les sources qui permettent de retracer l'histoire de ces collaborations entre l'architecte et les artistes, mais aussi celle des œuvres d'art depuis leur réalisation. Parmi elles figurent les archives que l'exemple de Jean-Claude Dondel donne l'occasion d'évoquer plus particulièrement.

Shortly after the end of World War II, the French State created an administration under the authority of the Ministry of Education, which undertook the construction of educational facilities for schoolchildren and students of all levels. Between 1950 and 1970, this administration (the DESUS for Direction de l'Équipement scolaire universitaire et sportif) commissioned numerous architects for the realisation of new educational buildings. Amongst them was Jean-Claude Dondel (1904-1989), who designed several schools throughout France. These buildings are all characterised by the desire to see the $1 \%$ devoted to artistic decoration properly integrated into the architecture. To understand the history of this collaboration between architects and artists, as well understanding the works of art from the outset of their creation, various sources can be used. The archives left by Jean-Claude Dondel's are particularly useful for the exploration of this issue.

\section{INDEX}

Keywords : Lazlo Barta, Robert Couturier, Olivier Descamps, Jacques Despierre, Jean-Claude Dondel, Roger Dhuit, Marcel Gili, Max Ingrand, André Pierre, Georges Rohner, school architecture, $1 \%$ of the budget of a public building, archives

Mots-clés : Lazlo Barta, Robert Couturier, Olivier Descamps, Jacques Despierre, Jean-Claude Dondel, Roger Dhuit, Marcel Gili, Max Ingrand, André Pierre, Georges Rohner, architecture scolaire, $1 \%$ décoratif, archives

\section{AUTEUR}

\section{ANAÏS CARRÉ}

Diplômée du master d'archivistique (université Paris-8, 2016) et d'un master 2 recherche histoire de l'architecture (université Paris 1-Panthéon-Sorbonne, 2016) sous la direction d'Éléonore Marantz anaiscarre75012@gmail.com 